\title{
Controlling Cell Functions by Light
}

\author{
Guglielmo Lanzani, Maria Rosa Antognazza, Nicola Martino, Diego Ghezzi and Fabio Benfenati
}

\begin{abstract}
The capability to selectively affect vital functions in cell networks and sub-cell compartments in vitro or in vivo is a mission critical tool in neuroscience and medicine. Optical excitation is one of the main strategies used to achieve high spatial and temporal resolution. In the following we describe recent results and future approaches of cell photostimulation mediated by organic semiconducting polymers.
\end{abstract}

\section{INTRODUCTION}

The first report in scientific literature of the use of light to modulate cell activity dates back to $1891[1]$ when the French physicist Jacques-Arsene d'Arsonval demonstrated that it is possible to excite muscular tissue upon illumination. In 1941 Arvanitaki and Chalazonitis [2] were able to observe that light is able both to excite and inhibit neural activity in ganglion cells of the marine mollusk Aplysia californica. These studies were carried on twenty years later by Fork, who reported [3] in 1971 a pioneering experiment demonstrating that laser radiation, both in the blue and in the green, is able to stimulate Aplysia neurons and it can be used to map cellular interconnections. After these initial isolated reports, in recent years a variety of different techniques, all exploiting interaction of light with biological systems, have been demonstrated, thus contributing to a resurgence of interest in the optical approach. Other than direct stimulation of biological tissues, light-sensitive proteins and photoactive molecules, materials, and devices have been designed to improve efficiency and flexibility of optical excitation [4].

Use of light as a stimulation tool allows targeting single cells and even discrete regions of a single cell in a temporally and spatially precise manner, offering at the same time the opportunity to exploit different excitation geometries. Moreover, it allows decoupling recording from stimulation, and it avoids the physical contact between the stimulation source and the target cell, thus escaping problems related to the contact impedance and more generally to biocompatibility issues.

Direct optical excitation [5] of cell activity has been broadly reported in literature. Different excitation wavelengths have been employed, spanning from the UV, to the visible and to the NIR spectral range. Two-photon irradiation with a pulsed infrared laser is generally preferred, since it yields good tissue penetration due to the low extinction coefficient for long wavelength radiation, and it

G. L. is with the Center for Nano Science and Technology @PoliMi, Istituto Italiano di Tecnologia, Milano 20133, Italy, and with the Dept. of Physics, Politecnico di Milano, Milano 20133, Italy (0039 022399 9872; fax: 0039022399 9867; e-mail: Guglielmo.lanzani@,iit.it).

M. R. A. and N. M. are with the the Center for Nano Science and Technology@PoliMi, Istituto Italiano di Tecnologia, Milano 20133, Italy.

D. G. and F. B. are with the Department of Neuroscience and Brain Technologies, Istituto Italiano di Tecnologia, Genova 16163, Italy. confines the stimulus locus to a single focal plane. In these cases excitation usually follows from local disruption of the plasma membrane or of some internal organelle, but the production of reactive oxygen species (ROS) has been also proposed. The mechanism by which ROS generation results in cell polarization most likely involves inhibition of voltagegated $\mathrm{K}^{+}$-channels.

Infrared neural stimulation (INS) [6] uses infrared light pulses to modulate neuronal activity. INS relies on the high absorption of infrared light by water, which is abundantly present in biological tissue. A short pulse of infrared light leads to a local transient rise in temperature. This local heating reversibly alters the electrical capacitance of the plasma membrane, resulting in cell depolarization; however, also activation of temperature-sensitive ion channels has been demonstrated to play a role. INS facilitates long-lasting stimulation of a single nerve, without affecting neighboring structures; moreover, it has also been proven capable of inhibiting neuron firing and action potential transmission. However, it cannot provide single-cell spatial resolution or cell type specificity.

Light stimulation can also be used to biochemically activate a signaling molecule [7], such as a neurotransmitter or intracellular calcium, which was inert due to the presence of photocleavable shielding group. The initially inactive compound becomes functional when the shielding group is cleaved through photolysis by illumination with a specific wavelength. Subsequently, with its active region exposed, the compound can act on its cellular target. Caged glutamate, GABA, calcium and other compounds have successfully been applied to modulate neuronal activity. These techniques suffer however the main limitation that the cleaving process is irreversible and, once the functional compound has been activated, it cannot be easily silenced.

The discovery of light-sensitive ion channels from algae was the starting point for the development of a novel photostimulation method, termed optogenetics [8]. In this technique, a gene coding for light-activated ion channels or pumps, such as the excitatory channel-rhodopsins and the inhibitory halorhodopsins, is introduced into the tissue. The gene construct can be delivered in a variety of ways, including electroporation, transfection, or viral vectors. Subsequently, the expression of the exogenous protein renders cells light-sensitive. Inclusion of cell-type specific promoters can ensure that expression of the light-activated protein is restricted to the desired cell-type. Since its initial discovery, optogenetics research has generated a whole range of suitable proteins, which are activated optimally by various stimulus wavelengths, and display diverse properties regarding ion selectivity and kinetics.

Neuronal activity can be also optically modulated by application of a photoisomerizable molecule [9], called a 
photoswitch, which binds to a native ion channel or receptor, making it light sensitive. When properly attached, the conformational change of the photoswitch, resulting from photon absorption, exerts force on the ion channel causing its opening. Other types of photoswitches can regulate the activity of ligand-gated channels or receptors. In this case photoisomerization of the photoswitch leads to delivery/removal of the ligand to its binding site on the channel or receptor. Photoswitches can be applied both in a genetically targeted and non-genetic fashion and their target proteins can be endogenous and/or exogenous. Photoswitches have successfully been used to regulate voltage-gated $\mathrm{K}^{+}$ channels, acetylcholine receptors and kainate-type glutamate receptors.

Finally, several exogenous techniques [10] have been also proposed, based on the use of photoactive materials placed externally, in the close proximity of the cell, and able to transduce light excitation into an electrical, chemical, or thermal stimulus. Among reported transducers are inorganic nano- and micro-particles and silicon-based interfaces.

Here we describe the approach based on interfacing organic semiconductors with living cells.

\section{NEURONS AND ASTROCYTES}

\section{A. Organic semiconductors}

Organic semiconductors are based on carbon atoms. Due to extended $\pi$-electron delocalization they efficiently absorb light in the visible and near UV range and show decent electrical transport properties. Light emission in LEDs is perhaps the most successful application so far, but many other applications are under investigation, e.g. light detection, photovoltaic energy conversion and printed electronics, to name a few. The fundamental observation that promotes organic semiconductors as the material of choice for bioelectronics is their structural kinship to proteins, carbohydrates and nucleic acids. From a structural point of view, organic semiconductors can have a number of key enabling features for bio electronics, such as being biocompatible, biodegradable, soft and conformable. On the functional side, they can support electronic as well as ionic transport, and can be easily functionalized to enable specific excitation, probing and sensing capabilities. Among the huge available library of material, poly(3-hexylthiophene-2,5diyl), or P3HT, is one of the most investigated semiconducting polymer in the field of organic electronics. When mixed with an electron acceptor like PCBM (phenylC61-butyric acid methyl ester), it is able to efficiently generate charges from absorbed photons in the blue/green region of the visible spectrum.

\section{B. Neurons}

Cultures of primary rat hippocampal neurons can be prepared onto both P3HT:PCBM blends and pure P3HT films coated with the adhesion molecule poly-L-lysine, with no difference in vitality up to 28 days in vitro with respect to cultures on control substrates. Following this observation, in 2011 it was shown that the photovoltaic blend P3HT:PCBM deposited on a transparent ITO conducting layer is able to elicit electrical activity in primary neurons grown on top of it upon photo-stimulation [11]. By recording membrane potentials by means of whole-cell patch-clamp under currentclamp configuration, it was shown that a $20 \mathrm{~ms}$ green light pulse $\left(532 \mathrm{~nm}, 10-15 \mathrm{~mW} \mathrm{~mm}^{2}\right.$ ) (where P3HT is strongly absorbing) evoked the generation of action potentials in neurons with great reproducibility. The response time was very rapid (around ten milliseconds to reach the peak of the spike) and the photo-stimulation process was highly spatially selective: if the light spot was moved just outside the cell body, no significant activity could be elicited in the neuron. The photophysics of the photovoltaic blend used in this study suggest a role for bulk charge photo-generation in the excitation mechanism. Moreover, it has been found that the same transduction process of light pulses into neuronal electrical activity can also be obtained by using a neat P3HT film (deposited on ITO)[12]. The actual transduction mechanism is however still unknown.

\section{Astrocytes}

Astroglial ion channels are fundamental molecular targets in the study of brain physiology and pathophysiology. Novel tools and devices intended for stimulation and control of astrocytes ion channel activity are therefore highly desirable. The study of the interactions between astrocytes and biomaterials is also essential to control and minimize reactive astrogliosis, in view of the development of implantable functional devices. Experiments on astrocytes offer a chance to broaden the scope of the cell stimulation by polymer photoexcitation mechanism and to demonstrate a universal value. The successful growth of rat primary neocortical astrocytes on the top of a light sensitive, organic polymer film was recently reported [13]; by means of patch-clamp analyses, the effect of the visible light stimulation on membrane conductance was investigated. Photoexcitation of the active material causes a significant depolarization of the astroglial resting membrane potential: the effect is associated with an increase in whole-cell conductance at negative potentials. The magnitude of the evoked inward current density is proportional to the illumination intensity. Biophysical and pharmacological characterization suggests that the ion channel mediating the photo-transduction mechanism is a chloride channel, the $\mathrm{ClC}-2$ channel. These results open interesting perspectives for the selective manipulation of astrocyte bioelectrical activity by noninvasive, label-free, organic-based, photostimulation approaches. In addition it suggests that the polymer interface may display specificity in the excitation path, addressing a particular ion channel.

\section{CELl LineS}

Using neurons or astrocytes is demanding in terms of preparation, required specific know-how and specific facilities. On the other side, the material scientist willing to explore the fascinating bio-organic interface can hardly simulate the real situation with artificial means. A good compromise is to use cell lines. These are easy to handle, require less care in the maintenance and growth and allow limiting the use of animal, yet providing a good testing sample. 

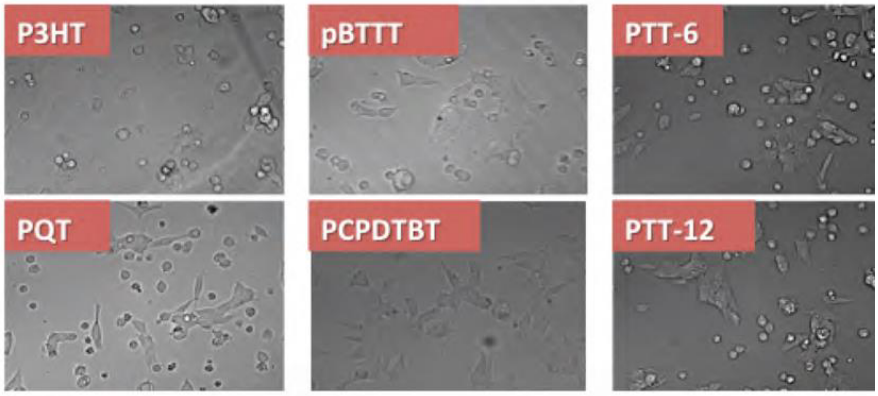

Figure 1. HEK-293 cells cultured on different polymer substrates: region-regular poly(3-hexylthiophene) (P3HT); poly(2,5-bis(3-hexadecylthiophen-2-yl)thieno[3,2-

b]thiophene) (pBTTT); poly-3,3"-dihexyl-2,2':5',2"terthiophene (3,3"-DHTT) (PTT-C6); poly[5,5'-bis(3-alkyl-2thienyl)-2,2'-bithiophene] (PQT); poly[2,6-(4,4-bis-(2ethylhexyl)-4H-cyclopenta[2,1-b;3,4-b']-dithiophene)-alt-4,7(2,1,3-benzothiadiazole)] (PCPDTBT); 3,3''-didodecyl2,2':5',2''-terthiophene (PTT-C12). All tested tiophenebased materials represent good substrates for cell seeding and culturing.

The photo induced stimulation on cell lines can be tested in the bio-organic interface as a photo induced depolarization or hyper polarization of the plasma membrane. The drawback is that cell lines usually have a limited number of ion channels, with a lower resting membrane potential, resulting into small modulation signals with respect to neurons. In addition, the action potential is obviously missing. In spite of this, the study of materials and architectures for cell photoexcitation can be carried out extensively.

HEK-293 (Human Embryonic Kidney 293) cells are one of the most used cell lines in biology. Given their small size, their low endogenous ionic currents and ease of transfection, they represent an invaluable tool in neuroscience research, for example in the study of expression and electrophysiology of ionic channels. Fig. 1 shows optical images of HEK-293 cells cultured on different polymer thin films, all based on thiophene units. To promote cellular adhesion to the substrate, the polymeric films are pre-treated with a coating of fibronectin, a protein of the extracellular matrix that binds to the integrins on the cell membrane. Fig. 2A shows a typical micrograph of an HEK-293 cell cultured on a polymer film, as it is approached by the patch electrode, which is used to measure the electrical potential across the cell membrane.

Light pulses absorbed by the active material elicit a response in the cell as a transient variation of its membrane potential. In Fig. 2, a typical response for a $200 \mathrm{~ms}$ pulse of blue light (excitation wavelength $\lambda=475 \mathrm{~nm}$, excitation density $\left.I=57 \mathrm{~mW} / \mathrm{mm}^{2}\right)$ is reported.

Upon illumination, the membrane potential depolarizes, i.e., it varies from its resting value (in this case about -41 $\mathrm{mV}$ ) towards more positive value. The depolarization is however transient, reaching a peak after few tens of milliseconds; then the potential tends towards less positive values, showing at the end a hyperpolarization signal. Upon switching off the light (in this case after $200 \mathrm{~ms}$ ), a reverse behavior is observed, with an overshoot of the membrane potential below its initial state (additional hyperpolarization) before the resting potential is ultimately recovered (after 800 $\mathrm{ms})$.
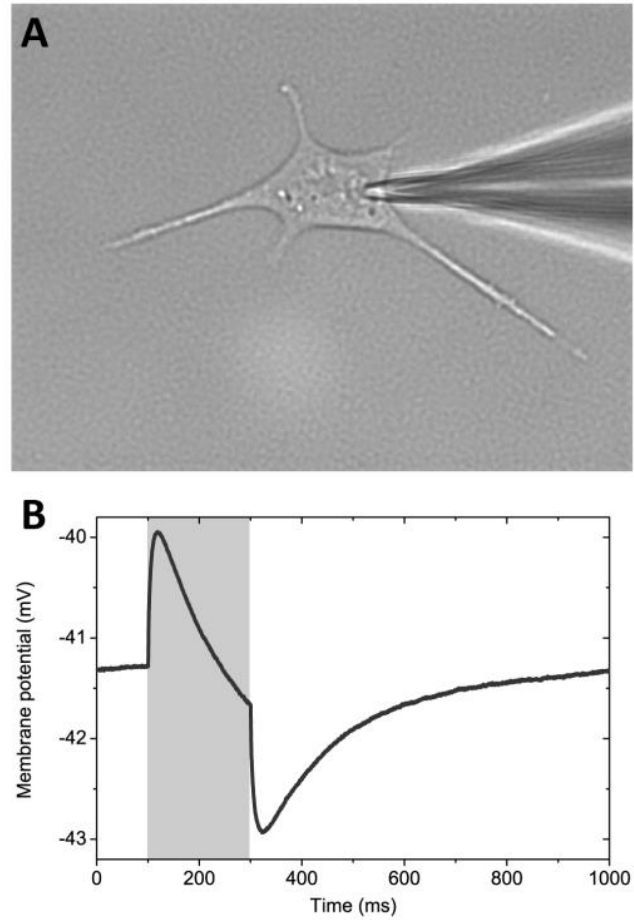

Figure 2. (A) A micrograph of an HEK-293 cell cultured on a thin film of P3HT, as it is approached by the patch electrode. (B) The variation of the membrane potential in a typical HEK-293 cell upon polymer photoexcitation (excitation wavelength $\lambda=475 \mathrm{~nm}, 200 \mathrm{~ms}$ long stimulus, excitation density $I=57 \mathrm{~mW} / \mathrm{mm}^{2}$ ).

\section{DISCUSSION}

Based on our preliminary work, different processes can be identified as main responsible for the transduction of light stimulus into cell activity manipulation and control, and more than one can be active in a specific interface.

Phototransduction operated by the polymer thin film can be sustained by: (i) a capacitive coupling between electronic charges accumulated at the surface of the active material and the ionic charges in the extracellular medium; and/or (ii) a net current flow established across the polymer/liquid interface; and/or (iii) thermally-mediated effects, which can modify the cell membrane electrical/mechanical/physical properties of the nearby cell, and eventually activate temperature-gated or mechanosensitive ion channels; and/or (iv) photo-activated reduction/oxidation reactions occurring at the interface and leading to modulation of $\mathrm{pH}$-regulated ion channels.

In spite of the uncertainty regarding the actual mechanism at work in the bio organic interface, the possibility to affect cell functions with light seems demonstrated. This opens up a wealth of applications. One, very straightforward, is the 
realization of a retinal implant. An active layer could be inserted under a defected retina (subretinal implant) or in contact with ganglion cells (epiretinal implant) in order to restore light sensitivity. Such an implant could be useful in blind patient whose natural photoreceptors are damaged or fully missing, like it is occurring in the final stage of Retinitis Pigmentosa and macular degeneration. The photo active layer could detect light and transduce it into a bio-electric signal that mimics the natural response, intercepting at some point the natural signal pathway to the optic nerve and brain. Promising results have been obtained in vitro [12] and are under investigation in vivo. This application, however, is just one of many for this emerging technology. The possibility to manipulate the cellular activity with a precise space and time resolution represents a key enabling technology for neuroscience and medicine. In vitro, it allows investigating neuronal networks and their functions with excellent spatial and temporal resolution in an easy, cheap and fast way, leading to new possibilities in the analysis and control of neural circuitry. In vivo, light addressable interfaces may have the potential of healing or controlling vital functions in the body other than sight.

\section{CONCLUSION}

Many fundamental processes and functions in living bodies are electrochemical in nature, most dealing with electro chemical dynamics at the cell membrane. Many diseases or disorders are associated with malfunctioning in such dynamics. Tools dedicated to selective and efficient cell stimulation offer a new and exciting chance for controlling, modifying and healing damaged tissues. The light addressable interfaces here discussed will represent in the next future valuable candidates for the toolbox of the neuroscientist and the medical doctor, allowing direct, minimally invasive, selective and rapid electrical control over cells.

\section{REFERENCES}

[1] J. A. D’Arsonval, C. R. Soc Biol. 1891, 43, 318

[2] A. Arvanitaki, N. Chalazonitis, Arch. Sci. Physiol. 1949, 3, 45.

[3] R. L. FORK, SCIENCE 1971, 171

[4] M. R. Antognazza et al., Adv. Mater., in press.

[5] P. Reece, K. Dholakia, R. Thomas, G. Cottrell, Neurosci. Lett. 433, 265 (2008).

[6] J. Wells, C. Kao, K. Mariappan, J. Albea, E. Jansen, P. Konrad, A. Mahadevan-Jansen, Opt. Lett. 30, 504 (2005); M. G. Shapiro, K. Homma, S. Villarreal, C.-P. Richter, F. Bezanilla, Nat. Commun. 3, 736 (2012); A. R. Duke, M. W. Jenkins, H. Lu, J. M. McManus, H. J. Chiel, E. D. Jansen, Sci. Rep. 3, 2600 (2013).

[7] K. Kandler, L. C. Katz, J. A. Kauer, Nat. Neurosci. 1, 119 (1998); J. Noh, R. P. Seal, J. A. Garver, R. H. Edwards, K. Kandler, Nat. Neurosci. 13, 232 (2010).

[8] E. S. Boyden, F. Zhang, E. Bamberg, G. Nagel, K. Deisseroth, Nature Neuroscience 8, 1263 (2005); S. Konermann, M. D. Brigham, A. Trevino, P. D. Hsu, M. Heidenreich, L. Cong, R. J. Platt, D. A. Scott, G. M. Church, F. Zhang, Nature 500, 472 (2013).

[9] A. Polosukhina, J. Litt, I. Tochitsky, J. Nemargut, Y. Sychev, I. De Kouchkovsky, T. Huang, K. Borges, D. Trauner, R. N. VanGelder and R. H. Kramer, Neuron 75, 271 (2012).

[10] J. Suzurikawa, M. Nakao, Y. Jimbo, R. Kanzaki, H. Takahashi, Sens. Actuators B 192, 393 (2014); J. Campbell, D. Singh, G. Hollett, S. M. Dravid, M. J. Sailor, J. Arikkath, Frontiers in Cellular Neuroscience DOI: $10.3389 /$ fncel.2014.00142 (2014).
[11] D. Ghezzi, M. R. Antognazza, M. Dal Maschio, E. Lanzarini, F. Benfenati \& G. Lanzani, Nat. Commun. 2, 166 (2011)

[12] D. Ghezzi, M. R. Antognazza, R. Maccarone, S. Bellani, E. Lanzarini, N. Martino, M. Mete, G. Pertile, S. Bisti, G. Lanzani, F. Benfenati, Nature Photonics 7, 400 (2013).

[13] V. Benfenati, N. Martino, M. R. Antognazza, A. Pistone, S. Toffanin, S. Ferroni, G. Lanzani, M. Muccini, Adv. Health Care Mater. 3, 392 (2014). 